International Journal of Pure and Applied Mathematics

Volume 115 No. $2 \quad 2017,361-367$

ISSN: 1311-8080 (printed version); ISSN: 1314-3395 (on-line version)

url: http://www.ijpam.eu

doi: 10.12732/ijpam.v115i2.13

\title{
FIXED POINTS OF CHATTERJEE AND CIRIC CONTRACTIONS ON AN $S$-METRIC SPACE
}

T. Phaneendra ${ }^{1}$, K. Kumara Swamy ${ }^{2}$

${ }^{1}$ Department of Mathematics

School of Advanced Sciences, VIT University

Vellore, 632014, Tamil Nadu, INDIA

${ }^{2}$ Department of Mathematics

Hyderabad Institute of Technology \& Management

Medchal District, Hyderabad, Telangana State, INDIA

Abstract: Unique fixed points are obtained for Chatterjee and Ciric contractions on an $S$-metric space, which are then shown to be $S$-contractive fixed points.

AMS Subject Classification: $54 \mathrm{H} 25$

Key Words: $S$-metric space, $S$-Cauchy sequence, Fixed point, $S$-contractive fixed point

\section{Introduction}

Let $X$ be a nonempty set. Sedghi et al [7] introduced an $S$-metric $S: X \times X \times$ $X \rightarrow[0, \infty)$ on $X$ satisfying the following conditions:

(S1) $S(x, y, z)=0$ if and only if $x, y, z \in X$ are such that $x=y=z$,

(S2) $S(x, y, z) \leq S(x, x, a)+S(y, y, a)+S(z, z, a)$ for all $x, y, z, a \in X$.

The pair $(X, S)$ is called an $S$-metric space. We obtain from Axiom (S2) that

$$
S(x, x, y)=S(y, y, x) \text { for all } x, y \in X .
$$

Received: $\quad$ February 23, 2017

Revised: $\quad$ May 23, 2017

Published: July 14, 2017

(c) 2017 Academic Publications, Ltd. url: www.acadpubl.eu

$\S_{\text {Correspondence author }}$ 
Definition 1.1. A sequence $\left\langle x_{n}\right\rangle_{n=1}^{\infty}$ in a $S$-metric space $(X, S)$ is said to be $S$-convergent, if there exists a point $x$ in $X$ such that $S\left(x_{n}, x_{n}, x\right) \rightarrow 0$ as $n \rightarrow \infty$.

Definition 1.2. A sequence $\left\langle x_{n}\right\rangle_{n=1}^{\infty}$ in a $S$-metric space $(X, S)$ is said to be $S$-Cauchy if $\lim _{n, m \rightarrow \infty} S\left(x_{n}, x_{n}, x_{m}\right)=0$.

Definition 1.3. The space $(X, S)$ is said to be $S$-complete, if every $S$ Cauchy sequence in $X$ converges in it.

The well-known infimum property of real numbers states that a nonempty and bounded set of real numbers has an infimum in $\mathbb{R}$. In particular,

Lemma 1.1. If $S$ is a nonempty subset of nonnegative real numbers, then $\alpha=\inf S \geq 0$ and $\lim _{n \rightarrow \infty} p_{n}=\alpha$ for some sequence $\left\langle p_{n}\right\rangle_{n=1}^{\infty}$ in $S$.

As an elementary application of Lemma 1.1, unique fixed points of a Chatterjee and Ciric- contractions are obtained in $S$-metric space. Further, the unique fixed points for these two contractions are shown to be $S$-contractive fixed points.

\section{Main Results}

Our first result is

Theorem 2.1. Let $f$ be a Chatterjee contraction on a complete $S$-metric space $(X, S)$ with the choice

$$
S(f x, f x, f y) \leq \alpha \max \{S(f x, f x, y), S(f y, f y, y)\} \text { for all } x, y \in X,
$$

where $0 \leq \alpha<1 / 3$. Then $f$ has a unique fixed point.

Proof. We divide the proof into various steps:

\section{Step 1 - Existence of the infimum}

Define $A=\{S(f x, f x, x): x \in X\}$. Then by Lemma 1.1, the infimum infimum $a$ of $A$ exists and is nonnegative.

\section{Step 2 - Vanishing infimum}

If $a>0$, writing $y=f x$ in (2.1) and using (1.1), we get

$$
S\left(f x, f x, f^{2} x\right) \leq \alpha \max \left\{S(f x, f x, f x), S\left(f^{2} x, f^{2} x, x\right)\right\}
$$




$$
\begin{aligned}
& \leq \alpha\left[S\left(f^{2} x, f^{2} x, f x\right)+S\left(f^{2} x, f^{2} x, f x\right)+S(x, x, f x)\right] \\
& =\alpha\left[2 S\left(f x, f x, f^{2} x\right)+S(f x, f x, x)\right]
\end{aligned}
$$

or

$$
\begin{aligned}
(1-2 \alpha) S\left(f x, f x, f^{2} x\right) & \leq \alpha S(f x, f x, x) \\
S\left(f x, f x, f^{2} x\right) & \leq\left(\frac{\alpha}{1-2 \alpha}\right) S(f x, f x, x)
\end{aligned}
$$

This implies that $0<a \leq \alpha a<a$, which is again a contradiction. Hence $a=0$.

\section{Step 3 - Existence of a sequence}

Hence, there exists a sequence $\left\langle x_{n}\right\rangle_{n=1}^{\infty}$ in $X$ such that

$$
S\left(f x_{n}, f x_{n}, x_{n}\right) \in A \text { for all } n=1,2,3, \ldots \text { and } \lim _{n \rightarrow \infty} S\left(f x_{n}, f x_{n}, x_{n}\right)=0 .
$$

\section{Step $4-\left\langle x_{n}\right\rangle_{n=1}^{\infty}$ is $S$-Cauchy}

In fact, by $(S 2)$ and (1.1), we have

$$
\begin{aligned}
S\left(x_{n}, x_{n}, x_{m}\right) \leq & S\left(x_{n}, x_{n}, f x_{n}\right)+S\left(x_{n}, x_{n}, f x_{n}\right)+S\left(x_{m}, x_{m}, f x_{n}\right) \\
= & 2 S\left(x_{n}, x_{n}, f x_{n}\right)+S\left(x_{m}, x_{m}, f x_{n}\right) \\
\leq & 2 S\left(x_{n}, x_{n}, f x_{n}\right)+S\left(x_{m}, x_{m}, f x_{m}\right)+S\left(x_{m}, x_{m}, f x_{m}\right) \\
& +S\left(f x_{n}, f x_{n}, f x_{m}\right) \\
= & 2\left[S\left(f x_{n}, f x_{n}, x_{n}\right)+S\left(f x_{m}, f x_{m}, x_{m}\right)\right] \\
& +S\left(f x_{n}, f x_{n}, f x_{m}\right) .
\end{aligned}
$$

Now, with $x=x_{n}$ and $y=x_{m},(2.1)$ gives,

$$
\begin{aligned}
S\left(f x_{n}, f x_{n}, f x_{m}\right) \leq & \alpha \max \left\{S\left(f x_{n}, f x_{n}, x_{m}\right), S\left(f x_{m}, f x_{m}, x_{n}\right)\right\} \\
\leq & \alpha \max \left\{\left[2 S\left(f x_{n}, f x_{n}, x_{n}\right)+S\left(x_{m}, x_{m}, x_{n}\right)\right]\right. \\
& {\left.\left[2 S\left(f x_{m}, f x_{m}, x_{m}\right)+S\left(x_{n}, x_{n}, x_{m}\right)\right]\right\} } \\
& =\alpha\left[2 S\left(f x_{n}, f x_{n}, x_{n}\right)+2 S\left(f x_{m}, f x_{m}, x_{m}\right)+2 S\left(x_{n}, x_{n}, x_{m}\right)\right]
\end{aligned}
$$

Inserting this in (2.3), we get

$$
\begin{gathered}
S\left(x_{n}, x_{n}, x_{m}\right) \leq(2 \alpha+1)\left[S\left(f x_{n}, f x_{n}, x_{n}\right)+S\left(f x_{m}, f x_{m}, x_{m}\right)\right] \\
+2 \alpha S\left(x_{n}, x_{n}, x_{m}\right)
\end{gathered}
$$


or

$$
(1-2 \alpha) S\left(x_{n}, x_{n}, x_{m}\right) \leq(2 \alpha+1)\left[S\left(f x_{n}, f x_{n}, x_{n}\right)+S\left(f x_{m}, f x_{m}, x_{m}\right)\right]
$$

so that

$$
S\left(x_{n}, x_{n}, x_{m}\right) \leq\left(\frac{2 \alpha+1}{1-2 \alpha}\right)\left[S\left(x_{n}, x_{n}, f x_{n}\right)+S\left(x_{m}, x_{m}, f x_{m}\right)\right]
$$

Applying the limit as $m, n \rightarrow \infty$ in this and using (2.2) we obtain that $\left\langle x_{n}\right\rangle_{n=1}^{\infty}$ is a $S$-Cauchy sequence in $X$.

\section{Step $5-S$-convergence}

Since, $X$ is $S$-complete, we find the point $p$ in $X$ such that

$$
\lim _{n \rightarrow \infty} x_{n}=p .
$$

\section{Step $6-S$-convergent limit as a fixed point}

Again repeatedly using $(S 2)$,

$$
\begin{aligned}
S(f p, f p, p) & \leq S\left(f p, f p, f x_{n}\right)+S\left(f p, f p, f x_{n}\right)+S\left(p, p, f x_{n}\right) . \\
& =2 S\left(f p, f p, f x_{n}\right)+S\left(p, p, f x_{n}\right) \\
& =2 S\left(f x_{n}, f x_{n}, f p\right)+S\left(f x_{n}, f x_{n}, p\right)
\end{aligned}
$$

Now, from (2.1) with $x=x_{n}$ and $y=p$, it follows that

$$
\begin{aligned}
S\left(f x_{n}, f x_{n}, f p\right) & \leq \alpha \max \left\{S\left(f x_{n}, f x_{n}, p\right), S\left(f p, f p, x_{n}\right)\right\} \\
& \leq \alpha\left[S\left(f x_{n}, f x_{n}, p\right)+S\left(f p, f p, x_{n}\right)\right]
\end{aligned}
$$

Substituting (2.6) in (2.5), we get

$$
\begin{aligned}
S(f p, f p, p) & \leq 2 \alpha\left[S\left(f x_{n}, f x_{n}, p\right)+S\left(f p, f p, x_{n}\right)\right]+S\left(f x_{n}, f x_{n}, p\right) \\
& =(2 \alpha+1) S\left(f x_{n}, f x_{n}, p\right)+2 \alpha S\left(f p, f p, x_{n}\right)
\end{aligned}
$$

In the limiting case as $n \rightarrow \infty$, this in view of (2.2) and (2.4) implies $S(f p, f p, p)=$ 0 or $f p=p$. Thus $p$ is a fixed point.

\section{Step 7 - Uniqueness of the fixed point}

Let $q$ be another fixed point of $f$. Then, (2.1) with $x=p$ and $y=q$ gives

$$
\begin{aligned}
S(p, p, q) & =S(f p, f p, f q) \\
& \leq \alpha \max \{S(f p, f p, q), S(f q, f q, p)\}
\end{aligned}
$$




$$
\begin{aligned}
& =\alpha \max \{S(p, p, q), S(q, q, p)\} \\
& =\alpha S(p, p, q)
\end{aligned}
$$

or

$$
(1-\alpha) S(p, p, q) \leq 0
$$

so that $p=q$. That is, $p$ is the unique fixed point of $f$.

Our next result is:

Theorem 2.2. Let $f$ be a Ciric-type contraction on a complete $S$-metric space $(X, S)$ such that

$$
\begin{gathered}
S(f x, f x, f y) \leq \alpha \max \{S(x, x, y), S(f x, f x, x), S(f x, f x, y), \\
S(f y, f y, x), S(f y, f y, y)\}
\end{gathered}
$$

for all $x, y \in X$, where $0 \leq \alpha<1 / 3$. Then $f$ has a unique fixed point.

A unique fixed point $p$ for (2.7) is obtained, similar to the previous proof and is omitted here.

\section{S-Contractive Fixed Point}

The notion of a $G$-metric space was introduced by Mustafa and Sims in [1], as a generalization of a metric space. In this setting, contractive fixed points were introduced in [2]. For further study on this idea, one can refer to $[3,4,5,6]$.

Now, we have

Definition 3.1. Let $f$ be a self-map on an $S$-metric space $(X, S)$. A fixed point $p$ of $f$ is a contractive fixed point, if for every $x_{0} \in X$, the $f$-orbit $O_{f}\left(x_{0}\right)=\left\langle x_{0}, f x_{0}, \ldots, f^{n} x_{0}, \ldots\right\rangle$ converges to $p$.

We now show that the unique fixed point $p$ of Chatterjee contraction (2.1) is an $S$-contractive fixed point.

Proof. Writing $x=f^{n-1} x_{0}, y=p$ in (2.1), we get

$$
\begin{aligned}
& S\left(f^{n} x, f^{n} x, p\right)=S\left(f^{n} x, f^{n} x, f p\right) \\
& \leq \alpha \max \left\{S\left(f^{n} x, f^{n} x, p\right), S\left(f p, f p, f^{n-1} x\right)\right\} \\
& =\alpha \max \left\{S\left(f^{n} x, f^{n} x, p\right), S\left(f^{n-1} x, f^{n-1} x, f p\right)\right\}
\end{aligned}
$$




$$
\begin{aligned}
& \leq \max \left\{S\left(f^{n} x, f^{n} x, p\right),\left[2 S\left(f^{n-1} x, f^{n-1} x, f^{n} x\right)+S\left(f p, f p, f^{n} x\right)\right]\right\} \\
& =\max \left\{S\left(f^{n} x, f^{n} x, p\right),\left[2 S\left(f^{n-1} x, f^{n-1} x, f^{n} x\right)+S\left(f^{n} x, f^{n} x, p\right)\right]\right\} \\
& \leq \alpha\left[2 S\left(f^{n-1} x, f^{n-1} x, f^{n} x\right)+S\left(f^{n} x, f^{n} x, p\right)\right]
\end{aligned}
$$

or

$$
\begin{aligned}
(1-\alpha) S\left(f^{n} x, f^{n} x, p\right) & \leq 2 \alpha S\left(f^{n-1} x, f^{n-1} x, f^{n} x\right) \\
S\left(f^{n} x, f^{n} x, p\right) & \leq\left(\frac{2 \alpha}{1-\alpha}\right) S\left(f^{n-1} x, f^{n-1} x, f^{n} x\right)
\end{aligned}
$$

Proceeding the limit as $n \rightarrow \infty$ in (3.1), we get $S\left(f^{n} x, f^{n} x, p\right) \rightarrow 0$. Thus $f^{n} x_{0} \rightarrow p$ for each $x_{0} \in X$. Thus $p$ is a $S$-contractive fixed point of $f$.

We finally show that the unique fixed point $p$ of Ciric contraction (2.7) is an $S$-contractive fixed point as follows:

Proof. Writing $x=f^{n-1} x_{0}, y=p$ in (2.7), and then using (1.1), we get

$$
\begin{aligned}
& S\left(f^{n} x, f^{n} x, f p\right)=S\left(f^{n} x, f^{n} x, p\right) \\
& \leq \alpha \max \left\{S\left(f^{n-1} x, f^{n-1} x, p\right), S\left(f^{n} x, f^{n} x, f^{n-1} x\right), S\left(f^{n} x, f^{n} x, p\right),\right. \\
& \left.S\left(f p, f p, f^{n-1} x\right), S(f p, f p, p)\right\} \\
& \leq \alpha \max \left\{S\left(f^{n-1} x, f^{n-1} x, p\right), S\left(f^{n} x, f^{n} x, f^{n-1} x\right), S\left(f^{n} x, f^{n} x, p\right)\right\} \\
& \leq \alpha \max \left\{\left[2 S\left(f^{n-1} x, f^{n-1} x, p\right)+S\left(p, p, f^{n} x\right)\right]\right. \\
& \left.S\left(f^{n} x, f^{n} x, f^{n-1} x\right), S\left(f^{n} x, f^{n} x, p\right)\right\} \\
& \leq \alpha\left[2 S\left(f^{n-1} x, f^{n-1} x, p\right)+S\left(f^{n} x, f^{n} x, p\right)\right]
\end{aligned}
$$

or

$$
\begin{aligned}
(1-\alpha) S\left(f^{n} x, f^{n} x, p\right) & \leq 2 \alpha S\left(f^{n-1} x, f^{n-1} x, f^{n} x\right) \\
S\left(f^{n} x, f^{n} x, p\right) & \leq\left(\frac{2 \alpha}{1-\alpha}\right) S\left(f^{n-1} x, f^{n-1} x, f^{n} x\right)
\end{aligned}
$$

As $n \rightarrow \infty$ in $(3.1)$, we see that

$$
S\left(f^{n} x, f^{n} x, p\right) \rightarrow 0
$$

Thus $f^{n} x_{0} \rightarrow p$ for each $x_{0} \in X$. Thus $p$ is a $S$-contractive fixed point of $f$. 


\section{References}

[1] Z. Mustafa, B. Sims, A new approach to generalized metric spaces, Journal of Nonlinear and Convex Anal., 7, No. 2 (2006), 289-297.

[2] T. Phaneendra, K. Kumara Swamy, Unique fixed point in $G$-metric space through greatest lower bound properties, NoviSad J. Math., 43 (2013), 107-115.

[3] T. Phaneendra, S. Saravanan, On some misconceptions and chatterjee-type Gcontraction, Int. J. Pure Appl. Math., 109, No. 4, 789-797.

[4] T. Phaneendra, S. Saravanan, On G-contractive fixed points, Jnanabha, 46, 105-112.

[5] T. Phaneendra, S. Saravanan, Bounded orbits and G-contractive fixed points, Comm. Appl. Anal., 20, 441-457.

[6] S. Saravanan, T. Phaneendra, Fixed point as a G-contractive fixed point, Int. J. Appl. Engg. Res., 11, No. 1 (2016), 316-319.

[7] Shaban Sedghi, Nabi Shobe, Abdelkrim Aliouche, A generalization of fixed point theorems in S-metric spaces, Matematiqki Vesnik, 64, No. 3 (2012), 258-266. 
